\title{
JuSpace: A tool for spatial correlation analyses of magnetic resonance imaging data with nuclear imaging derived neurotransmitter maps
}

\author{
Juergen Dukart ${ }^{1,2}$ ( ) | Stefan Holiga ${ }^{3}$ | Michael Rullmann ${ }^{4}$ | \\ Rupert Lanzenberger $^{5}$ (1) | Peter C. T. Hawkins ${ }^{6}$ (c) | Mitul A. Mehta ${ }^{6}$ | \\ Swen Hesse $^{4}$ | Henryk Barthel ${ }^{4}$ | Osama Sabri ${ }^{4}$ | Robert Jech ${ }^{7}$ | \\ Simon B. Eickhoff ${ }^{1,2}$
}

${ }^{1}$ Institute of Neuroscience and Medicine, Brain \& Behaviour (INM-7), Research Centre Jülich, Jülich, Germany

${ }^{2}$ Institute of Systems Neuroscience, Medical Faculty, Heinrich Heine University Düsseldorf, Düsseldorf, Germany

${ }^{3}$ Roche Pharma Research and Early Development, Roche Innovation Center Basel, F. Hoffmann-La Roche Ltd., Basel, Switzerland

${ }^{4}$ Department of Nuclear Medicine, University Hospital of Leipzig, Leipzig, Germany

${ }^{5}$ Department of Psychiatry and

Psychotherapy, Medical University of Vienna, Vienna, Austria

${ }^{6}$ Department of Neuroimaging, Institute of Psychiatry, Psychology \& Neuroscience, King's College London, London, UK

${ }^{7}$ Department of Neurology and Center of Clinical Neuroscience, Charles University, 1st Faculty of Medicine and General University Hospital, Prague, Czech Republic

\section{Correspondence}

Juergen Dukart, Institute of Neuroscience and Medicine - INM-7: Brain and Behaviour, Research Center Jülich Institute of Systems Neuroscience, Medical Faculty, Heinrich Heine University Düsseldorf Wilhelm-Johnen-Straße, 52425 Jülich, Germany.

Email: juergen.dukart@gmail.com

Funding information

Czech Ministry of Health, Grant/Award

Number: AZV NV19-04-00233; Horizon 2020

Framework Programme, Grant/Award

Number: 785907

\begin{abstract}
Recent studies have shown that drug-induced spatial alteration patterns in resting state functional activity as measured using magnetic resonance imaging (rsfMRI) are associated with the distribution of specific receptor systems targeted by respective compounds. Based on this approach, we introduce a toolbox (JuSpace) allowing for cross-modal correlation of MRI-based measures with nuclear imaging derived estimates covering various neurotransmitter systems including dopaminergic, serotonergic, noradrenergic, and GABAergic (gamma-aminobutric acid) neurotransmission. We apply JuSpace to two datasets covering Parkinson's disease patients (PD) and risperidone-induced changes in rsfMRI and cerebral blood flow (CBF). Consistently with the predominant neurodegeneration of dopaminergic and serotonergic system in $\mathrm{PD}$, we find significant spatial associations between rsfMRI activity alterations in PD and dopaminergic (D2) and serotonergic systems (5-HT1b). Risperidone induced $\mathrm{CBF}$ alterations were correlated with its main targets in serotonergic and dopaminergic systems. JuSpace provides a biologically meaningful framework for linking neuroimaging to underlying neurotransmitter information.
\end{abstract}

\section{KEYWORDS}

neuroimaging, positron emission tomography, neurotransmission, magnetic resonance imaging, Parkinson's disease, neuropharmacology

This is an open access article under the terms of the Creative Commons Attribution-NonCommercial License, which permits use, distribution and reproduction in any medium, provided the original work is properly cited and is not used for commercial purposes.

(c) 2020 The Authors. Human Brain Mapping published by Wiley Periodicals LLC. 


\section{1 | INTRODUCTION}

Magnetic resonance imaging (MRI)-derived measures are now commonly applied to study brain function and structure in health and disease (Bloudek, Spackman, Blankenburg, \& Sullivan, 2011; Bohanna, Georgiou-Karistianis, Hannan, \& Egan, 2008; Drysdale et al., 2017; Good et al., 2001). Voxel- and region-wise analyses are commonly applied to study associations between task-based (tbfMRI) and resting state (rsfMRI) MRI measures and observed symptoms, behavior or genetic information (van Erp et al., 2015; Meyer-Lindenberg \& Weinberger, 2006; Thompson et al., 2014; Thompson et al., 2020). RsfMRI measures provide replicable pathophysiological marker for various behaviors as well as different neurological and psychiatric conditions (Bernhardt, Bernasconi, Concha, \& Bernasconi, 2010; Holiga et al., 2019; Telesford et al., 2010). Despite this valuable information, biological and methodological limitations are imposed with respect to interpretation of the outcomes of voxel- and region-wise analyses.

From a methodological point of view, analyses of tbfMRI and rsfMRI are often limited by the rather low to moderate test-retest reliability of the commonly applied voxel- and atlas-based measures (Holiga et al., 2018). This low test-retest reliability and the resulting low signal-to-noise ratios impede constraints on the ability of fMRI to identify robust and replicable associations. Correspondingly, to date both tbfMRI and rsfMRI failed to achieve integration into routine clinical applications for the suggested indications (Lee, Smyser, \& Shimony, 2013; Leuthardt et al., 2018). Recent studies have shown that the overall spatial activity patterns (i.e., the relative within-subject activation of one region over another) of both tbfMRI and rsfMRI measures provide much more reliable marker as compared to standard voxel- and region-wise analyses (Dukart et al., 2018; Holiga et al., 2018). Making use of this higher reliability may therefore represent a viable way of improving the replicability of $f M R I$ applications.

From biology point of view, standard analyses of fMRI data focus on identification of voxel- or region-wise signals associated with a specific condition. While providing information about the spatial location of respective signals, such analyses do not allow drawing conclusions on potential neurophysiological mechanisms underlying the observed associations. To overcome this limitation, several recently published studies made use of spatial associations between underlying biology and observed imaging alterations by correlating ex vivo micro RNA spatial expression patterns with different imaging measures (Liu et al., 2019; Rizzo, Veronese, Expert, Turkheimer, \& Bertoldo, 2016; Selvaggi et al., 2018). The major idea behind such analyses is that disease- or drug-induced changes in imaging measures occur in association with availability of a specific tissue property (i.e., expression of a specific receptor) that is affected by the respective condition. For example, in a disease that is primarily associated with loss of dopaminergic neurons, one would expect strongest imaging changes in regions, which contain many of such neurons in healthy individuals. While promising, this approach also makes several assumptions that do not necessarily hold or are unknown for many of the underlying systems (Unterholzner et al., 2020). For example, correlations with
mRNA expression imply that the respective genes are transcribed and lead to measurable changes of tissue structure or function. A viable way of making use of this concept while avoiding these assumptions is by integration of positron emission tomography (PET) or single photon computed emission tomography (SPECT) derived tissue property maps. Recent advancements in PET and SPECT tracer development resulted in a variety of novel tracers that can reliably measure the availability of specific receptors but also functional aspects such as synthesis capacity across a variety of neurotransmitters (Beliveau et al., 2017; Lehto et al., 2015; Mawlawi et al., 2001; McCann et al., 2005; Smith et al., 1998). Such PET- and SPECT-derived maps provide a more direct measurement of specific tissue properties as compared to mRNA expression. In line with that, we have shown that MRI-derived spatial activity patterns induced by different drugs correlate with PET- and SPECT-derived information that are associated with the mechanism of action of respective compounds (Dukart et al., 2018).

Here we introduce the JuSpace toolbox allowing for spatial correlation of MRI-based or other imaging modalities with PET- and SPECT-derived maps covering a variety of neurotransmitter systems. To demonstrate its utility, we deploy the toolbox to rsfMRI data of Parkinson's disease (PD) patients on and off levodopa-a disease with devastating effects on multiple neurotransmitter systems, including major contributions from dopamine and serotonin (Booij et al., 1997; Pagano, Niccolini, Fusar-Poli, \& Politis, 2017) - as well as to cerebral blood flow data of healthy volunteers scanned on and off risperidone - an antipsychotic with a serotonergic and dopaminergic mechanism of action.

\section{2 | METHODS}

\subsection{Software description}

The main idea of JuSpace is to test if MRI-derived information is spatially structured in a way that reflects the distribution of specific biologically interpretable tissue properties as derived from PET and SPECT modalities. JuSpace is a comprehensive license-free toolbox (only for noncommercial use) for the integration of PET- and SPECTderived modalities with other brain imaging data. However, we do ask to cite the specific references for the PET and SPECT maps, which are used for the respective analyses. The references are provided in Table 1 . The currently released version is available at https://github.com/juryxy/JuSpace). JuSpace has been developed in the Matlab environment (Matlab 2017a or higher) and requires Statistical Parametric Mapping Software (SPM12, https://www.fil.ion. ucl.ac.uk/spm/software/spm12/) as well as the Matlab Statistics toolbox to be installed. More specifically, SPM routines are used to load, select and resize images into the atlas space. Gray matter tissue probability maps from SPM (TPM.nii) are used to account for spatial autocorrelation (if the respective option is selected). Stats toolbox is used to compute all correlational and multiple linear regression analyses. 
TABLE 1 Receptor maps included in the JuSpace toolbox

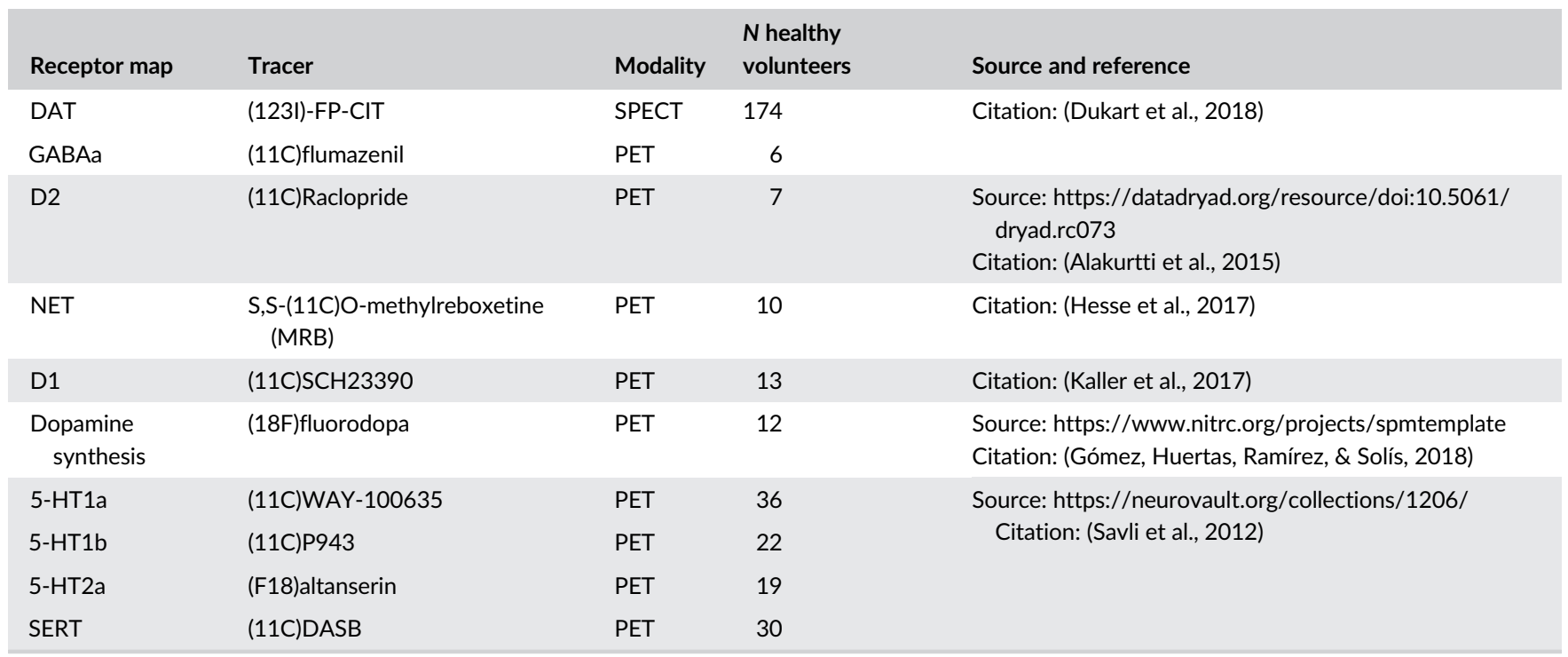

Abbreviations: DAT, dopamine transporter; NAT, noradrenaline transporter; PET, positron emission tomography; SERT, serotonin transporter; SPECT, single photon emission computed tomography.

JuSpace consists of a group of Matlab functions together with PET receptor maps covering various receptor systems. It provides a graphical user interface (Figure 1a) as well a direct call to the respective functions. There are no specific system requirements.

\section{2 | Included PET maps}

All PET and SPECT maps are free for noncommercial distribution and were previously published as described in Table 1 and in the release notes provided with the toolbox. All PET maps were derived from average group maps of different healthy volunteers and linearly rescaled to a minimum of 0 and a maximum of 100 :

$$
P E T_{\text {rescaled }}=100 * \frac{P E T-\min (P E T)}{\max (P E T-\min (P E T))}
$$

PET maps covering the following receptor types are included in the first release: 5-HT1a (serotonin 5-hydroxytryptamine receptor subtype 1a), 5-HT1b (5-HT subtype 1 b), 5-HT2a (5-HT subtype $2 \mathrm{a}$ ), D1 (dopamine D1), D2 (dopamine D2), DAT (dopamine transporter), F-DOPA (dopamine synthesis capacity), GABAa (gamma-aminobutric acid), NAT (noradrenaline transporter) and SERT (serotonin transporter) (for references see Table 1).

\section{3 | Workflow}

The analysis workflow starts with the user selecting the imaging (i.e., MRI) data to correlate with provided PET and SPECT maps. Either data for a single modality ("files 1" only) or data to generate a contrast between conditions ("files 1" and "files 2", i.e., patients vs. healthy controls or pre- vs. posttreatment data) are entered as input. The default atlas is the neuromorphometrics atlas from SPM12 (Friston et al., 1994) excluding all white matter and cerebrospinal fluid regions. A symmetric version of the atlas with bilateral regions of interest (left side flipped) is also included in the release. Neuromorphometrics atlas probability tissue labels were derived from the "MICCAI 2012 Grand Challenge and Workshop on MultiAtlas Labeling" (https://masi.vuse.vanderbilt.edu/workshop2012/ index.php/Challenge_Details). The atlas can be changed to any custom atlas using the "Select atlas" button. The atlas is used to extract mean regional values from the entered MRI modalities to be correlated with respective values from selected PET and SPECT maps. An atlas is needed as correlation of voxel-wise maps would result in highly inflated degrees of freedom. The number of distinct spatial features strongly depends on data smoothness but is typically in the range of several hundred or more distinct resolution elements (Mikl et al., 2008). In that sense, the default atlas with 119 regions provides a conservative estimate for the effective degrees of freedom. Next, the computing option is selected. Currently available options are:

1. Effect size between groups (computes Cohen's $d$ for each atlas region between files selected in list 1 and list 2)

2. Effect size of pair-wise differences (computes Cohen's $d$ for pairwise differences between files in list 1 relative to list 2)

3. Mean from list 1 (computes mean value per atlas region of all files from list 1)

4. List 1 each image (extracts mean value per atlas region for each file from list 1)

5. Compute individual $z$-score maps for each file in list 1 relative to list 2.

6. Computes pair-wise differences between list 1 and list 2 . 
(a)

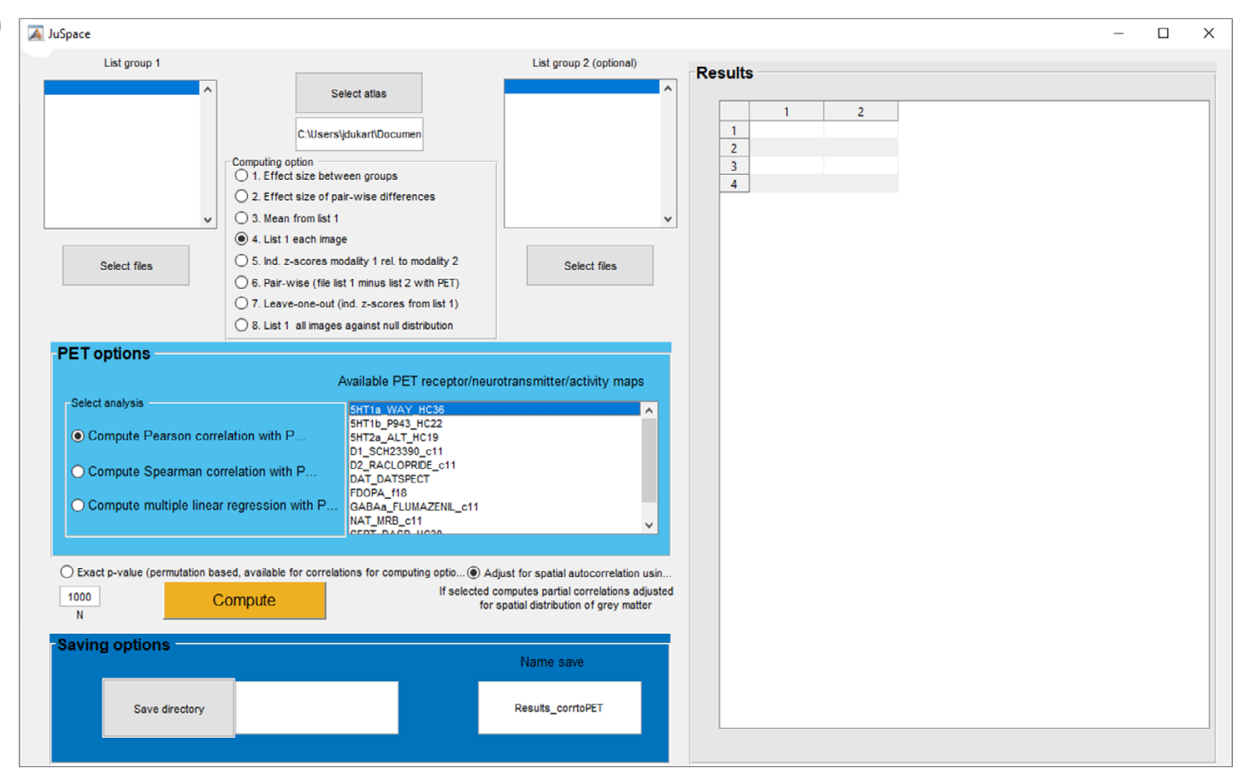

(b)

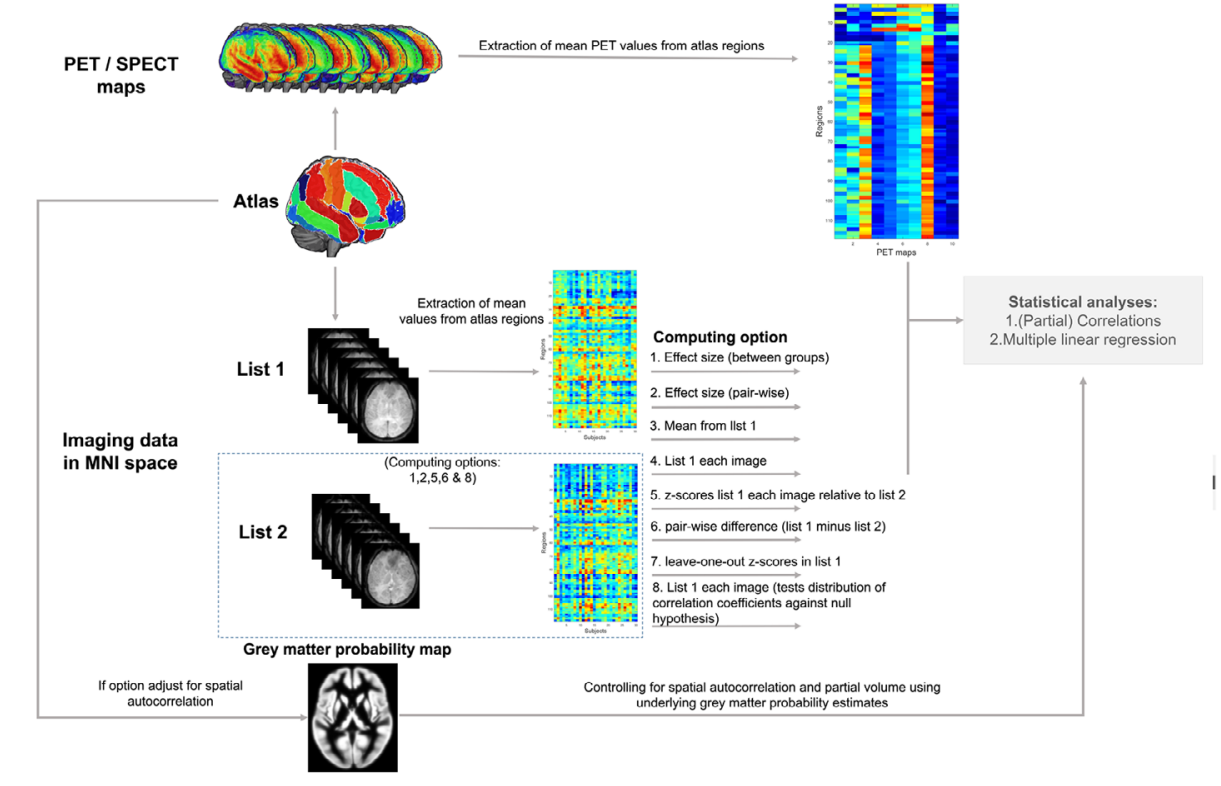

FIGURE 1 JuSpace toolbox, (a) User interface of the JuSpace toolbox, (b) Schematic work flow of the JuSpace toolbox
7. Computes leave-one-out $z$-scores maps for each file in list 1 relative to other files in list 1.

8. Extracts mean value per atlas region for each file from list 1 and compares correlation coefficients for all images against null distribution.

Further, the analysis type is selected (Pearson correlation, Spearman correlation or multiple linear regression). PET and SPECT maps can be selected by clicking on the respective name (multiple selection is supported, that is, hold control button during selection on a Windows machine). The currently available PET and SPECT maps are listed in Table 1.

Further, the option for exact permutation based $p$-value (only for computing options 1, 2, 5, and 6) can be selected (as described below). Additionally, the option is provided to adjust for spatial autocorrelation using the gray matter probability map TPM.nii from SPM12. The saving directory has to be specified using the "Save directory" button. The "Compute" button initiates the computation by calling the function "compute_DomainGauges" with the chosen computational parameters.

\subsection{Computational workflow}

All provided files as well as the selected PET maps are loaded into the atlas space as mean value per file and region (Figure $1 \mathrm{~b}$ ). Depending on the choice of the computing option, a spatial correlation or multiple linear regression is then computed between the selected PET maps and the extracted values as per selected computing option. In case adjustment the optional adjustment for spatial autocorrelation was selected (default), a partial spatial correlation is computed between both adjusting for local gray matter probabilities as 
estimated from TPM.nii provided with SPM12. In case of multiple linear regression, local gray matter probabilities are added as a covariate into the model. For correlation analyses, Fisher's z-transformed coefficients are provided as well as the original correlation coefficients. The distribution of Fisher's $z$ transformed correlation coefficients or regression coefficients for computing options 5-8 is compared against null distribution using one-sample t-tests. For group-level computing options $1-4$, the $p$-value is provided directly for the specific correlation /multiple linear regression analysis.

\section{5 | Permutation statistics}

An optional exact orthogonal permutation based p-value can be computed for correlational analyses (Pearson and Spearman) for computing options 1, 2, 5, and 6. The orthogonal permutation approach ensures that the shuffled labels vector is uncorrelated with the initial label vector providing a more valid null distribution (Aickin, 2010). The exact $p$-value is then computed using add-one discounting. For the within-subject designs (computing option 2 and 6), the permutations are performed by random switching of $50 \%$ of the data between files 1 and files 2 while maintaining pairwise associations. For the between-subject designs (computing options 1 and 5), the files from both groups are randomly permuted across files 1 and files 2 while maintaining the initial relative ratios of both groups in each permutation. For options 5 and 6 , the exact $p$-value is computed as the number of mean absolute correlation coefficients across permutations exceeding the observed mean absolute correlation coefficient.

\subsection{Correlation between PET and SPECT maps}

We computed Spearman correlations using computing option 4 with and without adjustment for auto-correlation to understand the interdependencies between all included PET and SPECT maps. Significant positive correlations were observed between most PET maps with strongest correlations of up to rho $=.89$ for GABAa and 5-HT2a and rho $=.88$ for DAT and SERT (all $p<.001$ ) (Figure 2). The only significant but weak negative correlation was observed between 5-HT1a and D2 (rho $=-0.19, p=.037$ ). To illustrate the utility of JuSpace we applied it to two datasets capturing disease- and drug-induced activity alterations as measured using rsfMRI.

\section{3 | APPLICATION EXAMPLE 1}

\subsection{Dataset}

To demonstrate the functionality of the JuSpace we applied it to an rsfMRI dataset of 30 PD patients scanned on and off levodopa as compared to 30 age and sex matched healthy controls (HC). A detailed description of the dataset as well of image acquisition and preprocessing is provided in Supplement 1. Fractional Amplitude of Low Frequency Fluctuations (fALFF) was computed as a measurement of local activity using the REST toolbox with default parameters (linear detrending and $0.01-0.08 \mathrm{~Hz}$ band-pass filtering). This measure was selected as it has been shown to most closely correlates with underlying metabolic activity as measured using glucose
FIGURE 2 Results of spatial correlation analyses between PET and SPECT derived neurotransmitter maps. The displayed numbers are the observed Spearman correlation coefficients. Significant correlations are highlighted by underlying ellipses. DAT, dopamine transporter; FDOPA, Fluorodopa; GABAa, $\gamma$-Aminobutyric acid type A; NAT, noradrenaline transporter; SERT, serotonin transporter

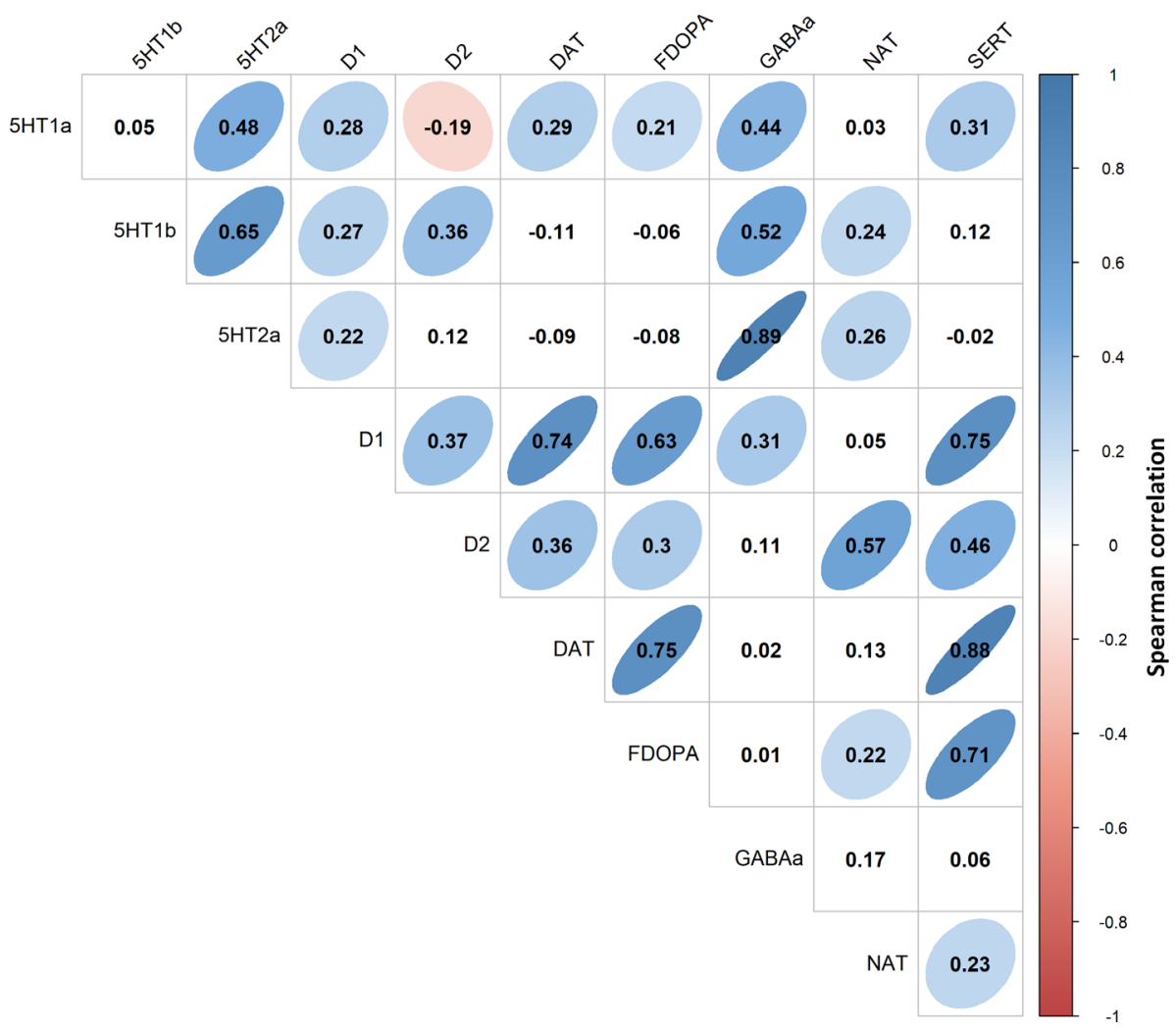


utilization as compared to other commonly used rsfMRI measures (Aiello et al., 2015).

\section{2 | Spatial correlation analyses}

We aimed to evaluate if fALFF alterations in PD patients on and off levodopa (individual z-scores) as compared to $\mathrm{HC}$ are correlated with specific neurotransmitter systems. For this, we used the JuSpace toolbox to compute Spearman correlation coefficients between respective measures (computing option 5) and the above PET maps included in the toolbox. We further aimed to test for the effects of levodopa on the fALFF maps. For this, we correlated the single subject pairwise differences between the fALFF maps with and without levodopa with the above PET maps (computing option 6). Exact permutation-based p-values (with 10,000 permutations) were computed for all analyses to test if the mean correlation coefficients observed across subjects are significantly different from the null distribution. All analyses were false discovery rate (FDR) corrected for the number of tests for each group comparison.

\section{3 | Voxel-wise analyses}

To compare the sensitivity and the information provided by the spatial correlation approach we additionally performed standard voxel-wise analyses in SPM12 comparing either PD patients on and off levodopa to $\mathrm{HC}$ (two-sample $t$-tests, including age and sex as covariates), or PD patients on levodopa to their off levodopa state (paired $t$-tests). The focus of the above spatial correlation analyses was on evaluating similarity of PD-related and drug-induced spatial patterns with specific PET maps. To visualize all regions showing strongest respective changes we applied a liberal voxel-wise threshold of $p<.05$ combined with a cluster threshold of 100 voxels. Additionally, we report all contrasts which survive classical voxel-wise whole-brain family-wise error correction ( $p<.05$ FWE corrected) for multiple comparisons.

\section{APPLICATION EXAMPLE 2}

\section{1 | Dataset and image processing}

In a second application example, we applied JuSpace to a CBF dataset of healthy volunteers $(N=21)$ scanned on placebo and on a low and high dose of the dopamine antagonist risperidone (0.5 and $2 \mathrm{mg})-\mathrm{a}$ serotonin and dopamine antagonist. Quantitative CBF maps in standard units ( $\mathrm{ml}$ blood/100 $\mathrm{mg}$ tissue/min) were computed from a pseudocontinuous Arterial Spin Labeling sequence using a proton density maps. The risperidone cohort is described in detail in Supplement 1 and in a previous publication (Hawkins et al., 2018).

Pre-processing of CBF data was performed using the Statistical Parametric Mapping (SPM12) software package (Friston et al., 1994). CBF data were first co-registered to individual structural T1 images. Structural scans were segmented and normalized into the Montreal Neurological Institute (MNI) space using the SPM Segment function. Deformation parameters derived from this normalization were then applied to the co-registered and gray matter masked CBF data to bring them into the MNI space. Additionally, smoothing with Gaussian kernel of $8 \mathrm{~mm}$ FWHM was applied prior to voxel-wise analyses.

\section{2 | Spatial correlation analyses}

For the risperidone dataset, we tested for the effects of high and low dose of risperidone as compared to placebo and to each other by computing Spearman correlation coefficients between respective within-subject pairwise differences (computing options 6). Exact permutation-based $p$-values (10,000 permutations) were computed for all analyses. All analyses were FDR corrected according to the number of tests for each group comparison.

\section{3 | Voxel-wise analyses}

We computed a within subject ANOVA to compare risperidone high dose, risperidone low dose and placebo conditions using pair-wise tcontrasts. Same voxel- and cluster-wise thresholds as for the PD dataset were applied.

\section{5 | IMPACT OF ATLAS CHOICE}

We further aimed to evaluate how the choice of an atlas affects the consistency of findings observed in the above spatial correlation analyses. For this, all spatial correlation analyses for application examples 1 and 2 that were computed using the Neuromorphometrics atlas were repeated using the also commonly applied automated anatomical labeling (AAL) atlas (Tzourio-Mazoyer et al., 2002). Consistency of the individual correlations observed with both atlases across subjects for the different neurotransmitter maps was evaluated using Cronbach's alpha as implemented in the ICC (intraclass correlation coefficient) function (" $\mathrm{C}-\mathrm{k}$ " option) in Matlab.

\section{6 | RESULTS}

\section{1 | Application example 1}

\subsection{1 | Results of spatial correlation analyses}

Individual fALFF alterations in PD patients off levodopa as compared to $\mathrm{HC}$ were significantly associated with spatial distribution of D2 
(a)

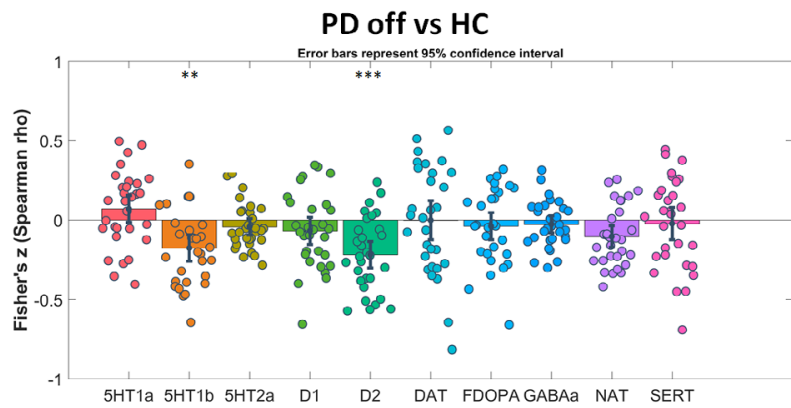

(b)

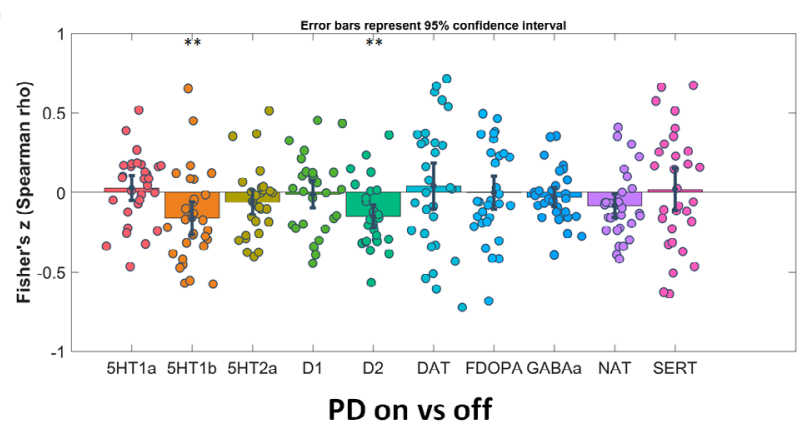

(c)

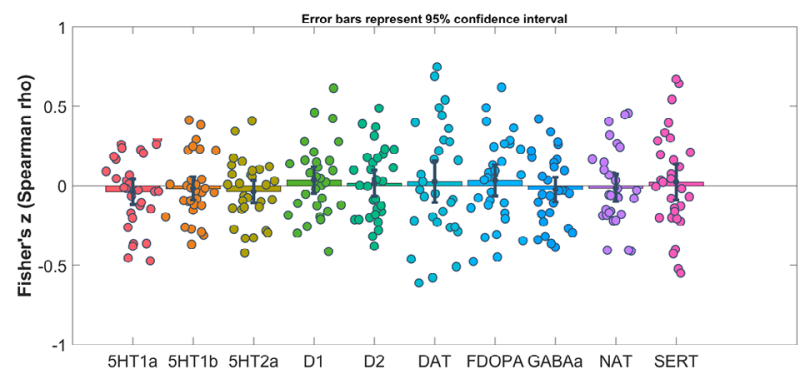

(d)

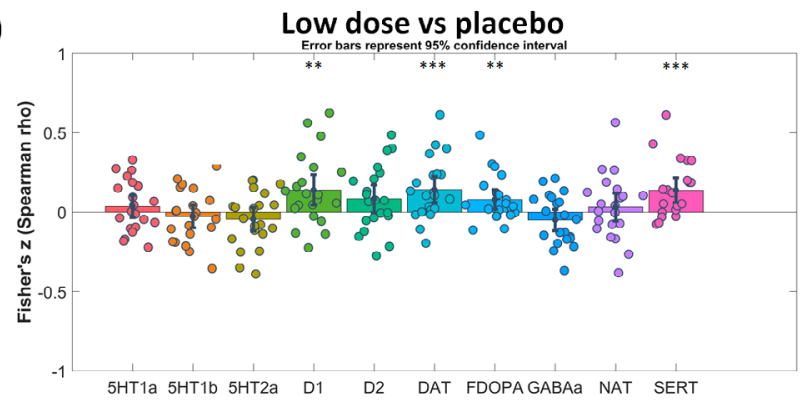

(e)

High dose vs placebo

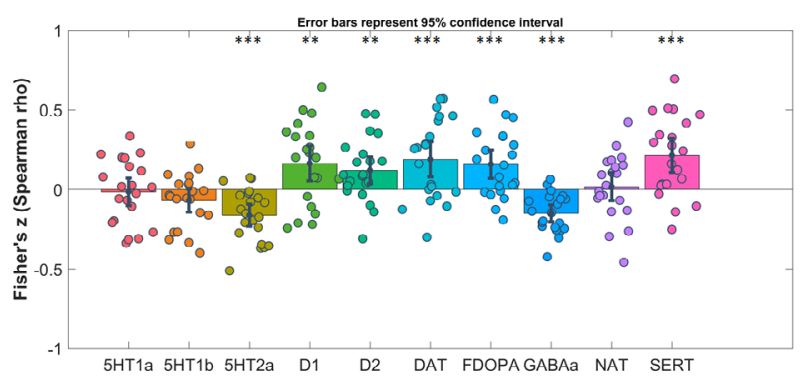

(f)

High dose vs low dose

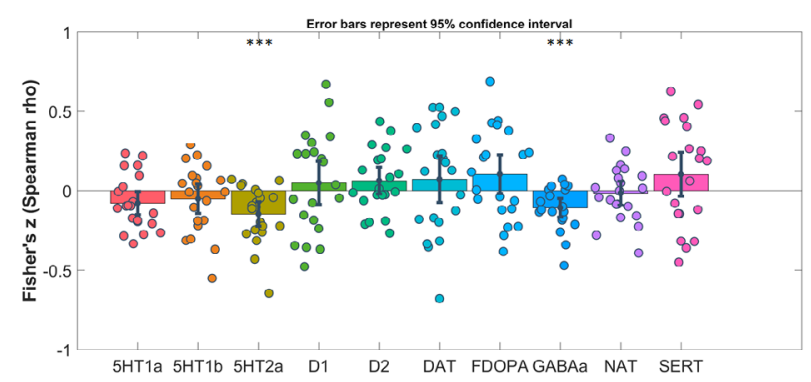

FIGURE 3 Results of spatial correlation analyses for PD and risperidone datasets. Fisher's z-transformed correlation coefficients with respective neurotransmitter maps are displayed for each subject and contrast. Error bars represent the parametric $95 \%$ confidence interval of the mean. DAT, dopamine transporter; FDOPA, Fluorodopa; GABAa, $\gamma$-Aminobutyric acid type A; HC, healthy controls; NAT, noradrenaline transporter; PD, Parkinson's disease; SERT, serotonin transporter

$(p<.001)$ and 5-HT1b $(p=.003)$ receptors as derived from healthy subjects (Figure 3a). Similarly, fALFF alterations in PD patients on levodopa were significantly associated with availability of D2 ( $p=.002$ ) and 5-HT1b receptors (both $p=.008$ ) (Figure 3b). There was no significant difference between levodopa on and off conditions (all $p>$.29) (Figure 3c).

\subsection{2 | Results of voxel-wise analyses}

In voxel-wise comparisons to HC, decreased fALFF was observed in $\mathrm{PD}$ on and off levodopa conditions in an extensive network covering predominantly prefrontal, parietal, cerebellar, basal ganglia, supplementary and primary motor regions (Figure 4a,b). Increased fALFF was observed primarily in temporal and orbitofrontal cortices. Decreased fALFF was observed in PD on levodopa as compared off levodopa in prefrontal, left temporal and right parietal cortices (Figure 4c). None of the effects survived whole-brain voxel-wise correction for multiple comparisons.

\section{2 | Application example 2}

\subsection{1 | Results of spatial correlation analyses}

CBF alterations induced by the low dose of risperidone as compared to placebo were significantly associated with D1 $(p=.002)$, DAT $(p<.001)$, F-Dopa $(p=.006)$ and SERT $(p<.001)$ maps (Figure 3d). CBF changes induced by the high dose of risperidone were significantly correlated with 5 -HT2a $(p<.001)$, D1 ( $p=.003)$, D2 $(p=.003)$, DAT $(p<.001)$, GABAa $(p<.001)$ and SERT $(p<.001)$ maps (Figure 3e). CBF changes with the high dose as compared to low dose of risperidone were significantly associated with $5-\mathrm{HT} 2 \mathrm{a}$ and GABAa receptor availability (both $p<.001$ ) (Figure 3f).

\subsection{2 | Results of voxel-wise analyses}

Increased CBF was observed in basal ganglia comparing high and low dose of risperidone to placebo (Figure 3d,e). Reduced CBF in low dose 
(a)

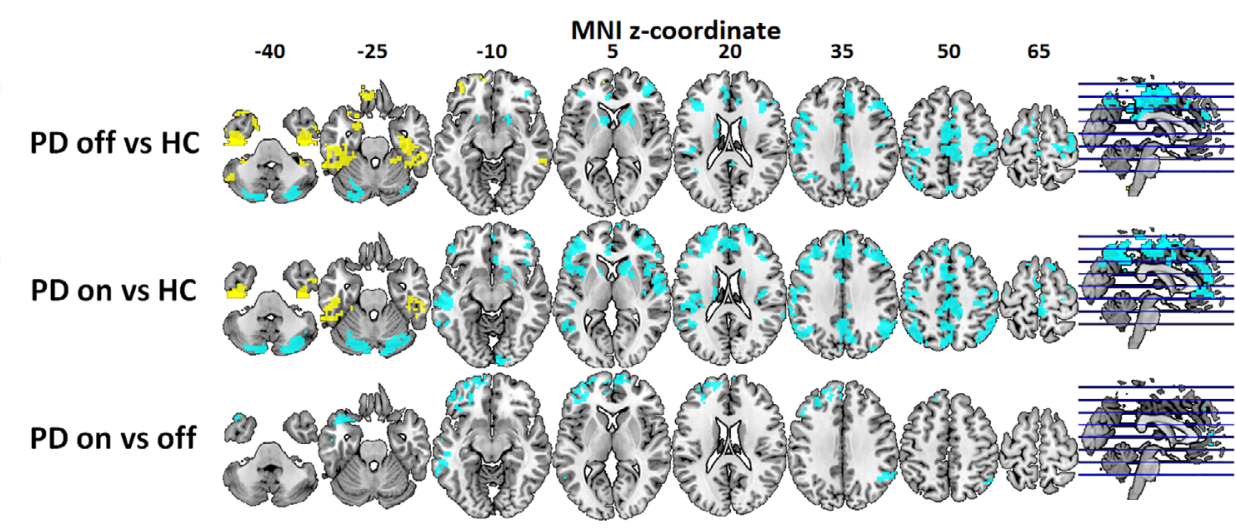

(d)

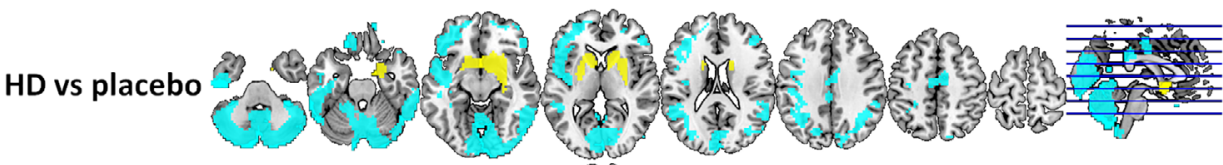

(e)
LD vs placebo

(f)
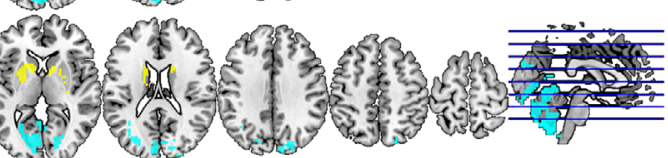

antw

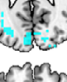

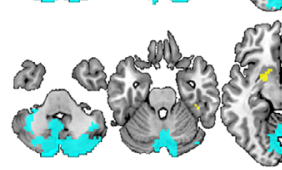

है।
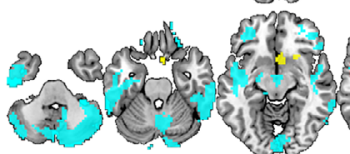

HD vs LD
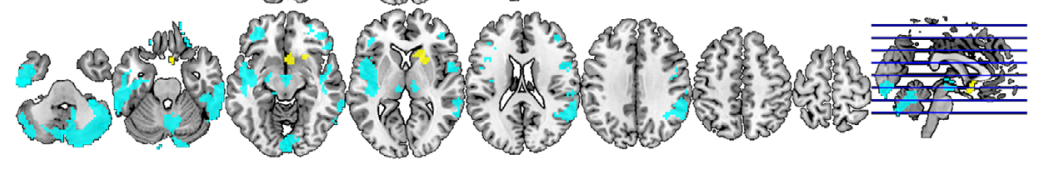

FIGURE 4 Results of voxel-

wise analyses for PD and risperidone datasets. Orange color indicates increased fALFF (for PD) or CBF (for risperidone) in the first mentioned condition/ group. Cyan color indicates decreased fALFF (for PD) or CBF (for risperidone) in the first mentioned condition/group. Montreal Neurological Institute (MNI) space z-coordinates are reported for each slice. HC, healthy controls; HD, high dose of risperidone; LD, low dose of risperidone; $\mathrm{PD}$, Parkinson's disease

as compared to placebo was predominantly restricted to occipital and cerebellar cortices. High dose associated changes were more extensive covering prefrontal, posterior cingulate, cerebellar temporal, parietal and occipital regions. High dose as compared to low dose of risperidone showed an increase in $\mathrm{CBF}$ in the right corpus striatum (Figure 3f). Reduced CBF was observed with high dose in prefrontal, temporal, occipital, parietal, and cerebellar regions. Only the reduced CBF in cerebellar and occipital regions in high dose as compared to placebo survived whole-brain voxel-wise correction for multiple comparisons.

\subsection{3 | Results for the impact of atlas choice}

A high median consistency ranging from ICC $=.83-.86$ was observed for the results obtained using the Neuromorphometrics and the AAL atlas for both application examples (Figure 5).

\section{7 | DISCUSSION}

Here we introduce the JuSpace Toolbox, an integrated system for the comparison of PET and SPECT derived neurotransmitter maps with other imaging modalities such as rsfMRI data. The software tests for associations between the imaging data of interest and a list of included PET and SPECT maps by computing correlations or multiple linear regressions.
JuSpace allows for an easy integration of neuroimaging data with PET-derived receptor maps. JuSpace is a user-friendly tool allowing user interface-based applications by researchers with limited programming experience as well as direct function calls. The choice of settings for the analyses is kept to a minimum. The toolbox further supports an easy integration of other atlases and other PET-derived information, provided they have the same resolution, by simply adding the respective maps in MNI space into the PET atlas directory. JuSpace is designed to test the hypotheses that the spatial structure of imaging alterations induced that is, by disease or drug is associated with the availability of a specific receptor across the brain. Besides direct correlation between the imaging data of interest and the available PET maps, the software supports simple between- and within-subject designs by computing effect sizes, $z$-scores and pair-wise differences. For both, between- and within-subject designs the toolbox provides more rigorous permutation based statistics. Usage of these exact statistics for both options is strongly recommended to avoid erroneous assumptions on data distribution or actual spatial degrees of freedom.

As compared to available imaging-genomic toolboxes correlating imaging information with the spatial information derived from few donors from the Allen Brain Atlas of postmortem mRNA expression JuSpace carries the advantage of making less assumptions about underlying biology (i.e., unknown transcription of respective mRNA into specific tissue properties) (Rizzo et al., 2016). It also allows for evaluation of spatial associations with PET-derived transmitter synthesis information that are only available from in vivo studies, that is, F-Dopa PET-derived dopamine synthesis capacity. 

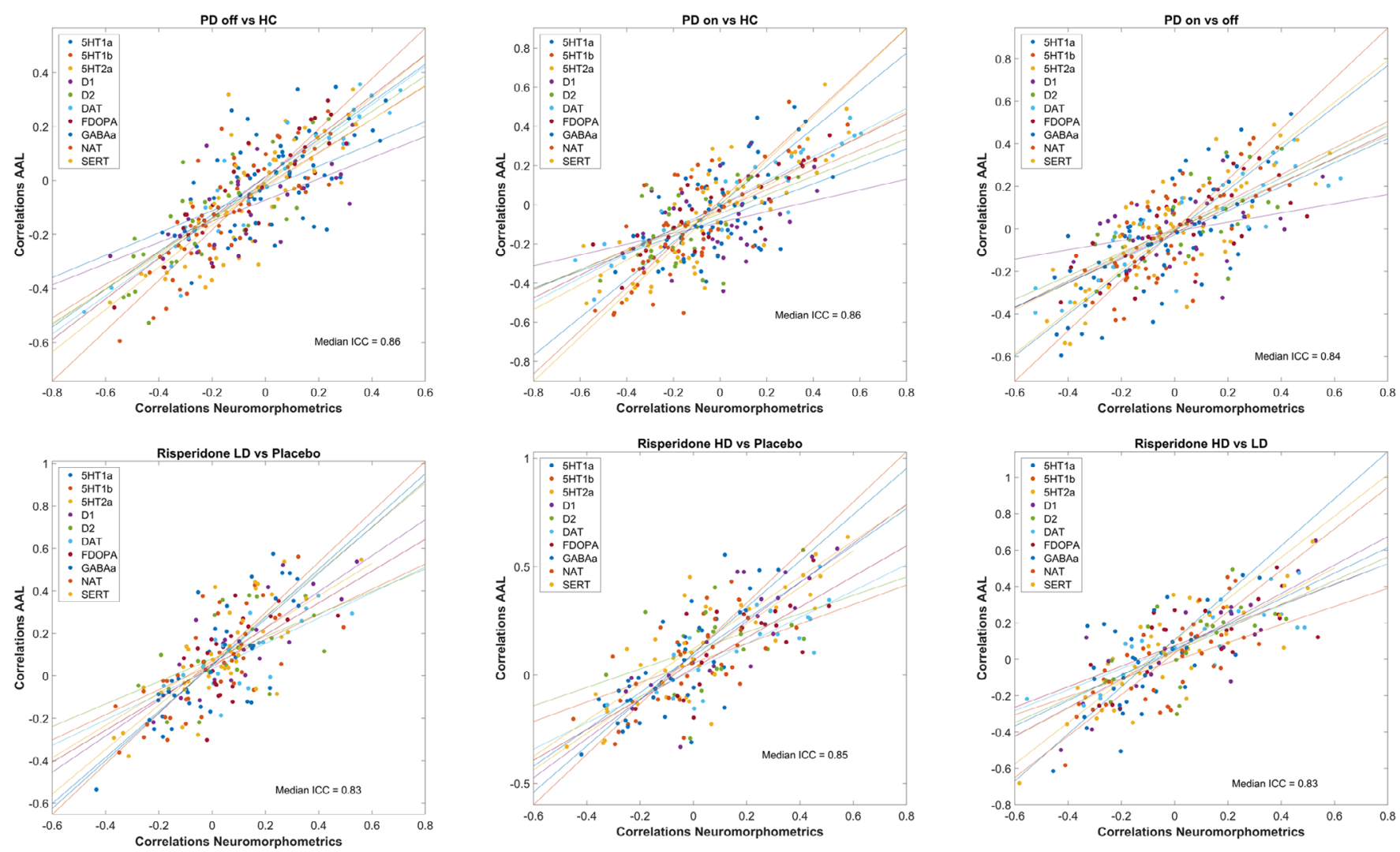

FIGURE 5 Results of consistency analyses comparing correlation coefficients obtained with the Neuromorphetrics and Automated Anatomical Labeling atlases. DAT, dopamine transporter; FDOPA, Fluorodopa; GABAa, $\gamma$-Aminobutyric acid type A; ICC, intraclass correlation coefficient; NAT, noradrenaline transporter; SERT, serotonin transporter

\section{8 | CONCLUSIONS FROM THE APPLICATION EXAMPLES}

Here we applied the JuSpace toolbox to two datasets covering disease related rsfMRI alterations in PD as well drug-induced CBF alterations in healthy controls. We show that rsfMRI activity alterations in PD on and off levodopa are closely associated with availability of D2 and 5 -HT1b receptors. These results are closely in line with the wellestablished affectedness of the dopaminergic and serotonergic systems in PD (Booij et al., 1997; Pagano et al., 2017). Furthermore, the consistency of the effects obtained with both scans in PD illustrates the robustness of findings obtained using spatial correlation analyses. We do not find significant associations between PET maps and fALFF differences between PD on and off levodopa. This is potentially explained by the effects being either subtle or not following the distribution of the specific receptor maps currently included in JuSpace.

Risperidone-induced brain activity alterations are associated with a variety of neurotransmitter systems including dopaminergic and serotonergic effects. In contrast to the highest affinity of risperidone to $5-\mathrm{HT} 2 \mathrm{a}$ followed by D2 the significant associations with the corresponding PET receptor maps only appeared with the high dose (Schotte et al., 1996). The strongest effects observed with low dose rather associate with DAT, SERT and D1 receptor maps. In our previous study (Selvaggi et al., 2018) we observed correlations with D2 receptors at both doses for the group averaged data. This study did not examine effects using other targets and did not evaluate individual differences. Importantly, the spatial correlation analysis relies on a direct translation of the effect observed on the specific receptor into the respective activity measurement, which may vary by drug and across subjects. Indeed, we see a substantial variation across subjects in the strength and direction of the observed correlations. This variation may reflect the individual susceptibility of specific receptor systems to the disease process or in case of drug data the strength of the pharmacodynamics response. Yet, ideally simultaneous PET and MRI studies are needed to provide a detailed insight into the mechanisms underlying this variation. The observed discrepancies suggest that the effects on different receptors may have different transfer function onto the observed CBF changes that is not directly associated with respective receptor affinities. This observation is in line with our previous work evaluating correlations with postmortem receptor expression (Dukart et al., 2018). Overall, while the observed correlations with serotonergic and dopaminergic system are in line with the known mechanism of action of risperidone the correlation with GABAa receptor appearing with the high dose may appear unexpected as there is no reported affinity of risperidone to the respective receptor. There are two potential explanations for this effect. We observe a 
very strong positive correlation between GABAa and 5-HT2a receptor availability. In that sense, the observed correlation with both receptors is likely due to the collinearity of both receptor systems making it difficult to dissociate the specific effects on one of the two systems. Another possible explanation for the observed correlation with GABAa may be in the reported interdependence of both systems with 5-HT2a activation resulting in inhibition of GABAa currents (Feng et al. 2001). Similar to that, the strong correlation of PET maps may also explain the observed correlation of the low dose changes with DAT, SERT and D1 as all of the PET maps are strongly correlated. These strong cross-correlations between different PET maps indicate that caution is required with respect to interpretation of observed associations as being specific to a particular receptor. Further analyses adjusting for cross-correlation of the PET receptors such as the multiple linear regression analyses option also provided with the toolbox may facilitate the interpretation in such cases.

Whole-brain corrected analyses of both datasets only revealed differences between high dose of risperidone and placebo. The results applying a more liberal threshold were rather diffuse covering a wide-range of regions making it difficult to interpret the findings with respect to any specific anatomical circuitries. In contrast, spatial correlation analyses reveal a higher sensitivity to both PD- and risperidone-induced changes and additionally provide a biologically meaningful interpretation of the observed effects. Our results therefore suggest the higher sensitivity of the spatial correlation approach to detect disease- and drug-related activity alterations as compared to standard voxel-wise analyses. The likely reasons for the higher sensitivity of the spatial correlation as compared to voxel-wise analysis is the substantially higher reliability of spatial activity profiles as compared to classical voxel- or region-wise fALFF and CBF measures (Holiga et al., 2018). In line with this notion of high test-retest reliability, we find a high consistency of all results with respect to the choice of the applied atlas. However, these results also demonstrate that the choice of the atlas may introduce some variability into the outcomes. It will be therefore important for future studies to systematically evaluate which atlas provide an optimum choice with respect to differentiation of regions with distinct neurotransmitter profiles. In this context, it will be also important to test the applicability and reproducibility of the introduced spatial correlation approach across different datasets.

Overall, the JuSpace toolbox allows for cross-modal evaluation of neuroimaging data alongside molecular imaging atlases as also illustrated in recent publications. The inclusion of PET and SPECT atlases for different neurotransmitter systems allows for biologically meaningful evaluation and interpretation of the spatial patterns. This is a flexible platform enabling inclusion of user-defined atlases and other imaging modalities. As such, it has a great potential to improve and simplify multi-modal brain imaging research.

\section{ACKNOWLEDGMENTS}

The PD study was supported by the Czech Ministry of Health, grant AZV NV19-04-00233. This open source software code was developed in part or in whole in the Human Brain Project, funded from the European Union's Horizon 2020 Framework Programme for Research and Innovation under the Specific Grant Agreement No. 785907 (Human Brain Project SGA2). The funders had no role in study design, data collection and analysis, decision to publish, or preparation of the manuscript.

\section{CONFLICT OF INTERESTS}

Stefan Holiga is current employee of F.Hoffmann-La Roche. Juergen Dukart is a former employee and currently consultant for F.Hoffmann-La Roche. Rupert Lanzenberger received travel grants and/or conference speaker honoraria within the last 3 years from Bruker BioSpin MR, Heel, and support from Siemens Healthcare regarding clinical research using PET/MR. He is a shareholder of BM Health $\mathrm{GmbH}$ since 2019. All authors report no conflicts of interest with respect to the work presented in this study.

\section{AUTHOR CONTRIBUTIONS}

Juergen Dukart wrote the manuscript. Juergen Dukart designed the overall study, wrote the toolbox performed all analyses and wrote the manuscript. Mitul A. Mehta, Peter C. T. Hawkins and Robert Jech contributed to the application examples. Stefan Holiga, Michael Rullmann, Rupert Lanzenberge, Swen Hesse, Henryk Barthel, and Osama Sabri contributed to creation of PET and SPECT maps. Simon B. Eickhoff contributed to the overall design of the study as well as to toolbox conceptualization. All authors reviewed and commented on the manuscript.

\section{DATA AVAILABILITY STATEMENT}

The JuSpace toolbox is available at https://github.com/juryxy/ JuSpace

\section{ORCID}

Juergen Dukart (D) https://orcid.org/0000-0003-0492-5644

Rupert Lanzenberger (D) https://orcid.org/0000-0003-4641-9539

Peter C. T. Hawkins (D) https://orcid.org/0000-0002-2256-1648

\section{REFERENCES}

Aickin, M. (2010). Invalid permutation tests. International Journal of Mathematics and Mathematical Sciences, 2010, 1-10.

Aiello, M., Salvatore, E., Cachia, A., Pappatà, S., Cavaliere, C., Prinster, A., ... Quarantelli, M. (2015). Relationship between simultaneously acquired resting-state regional cerebral glucose metabolism and functional MRI: A PET/MR hybrid scanner study. Neurolmage, 113, 111-121.

Alakurtti, K., Johansson, J. J., Joutsa, J., Laine, M., Bäckman, L., Nyberg, L., \& Rinne, J. O. (2015). Long-term test-retest reliability of striatal and extrastriatal dopamine D2/3 receptor binding: Study with $((11) C)$ raclopride and high-resolution PET. Journal of Cerebral Blood Flow and Metabolism, 35, 1199-1205.

Beliveau, V., Ganz, M., Feng, L., Ozenne, B., Højgaard, L., Fisher, P. M., ... Knudsen, G. M. (2017). A high-resolution in vivo atlas of the human brain's serotonin system. Journal of Neuroscience, 37, 2830-2836.

Bernhardt, B. C., Bernasconi, N., Concha, L., \& Bernasconi, A. (2010). Cortical thickness analysis in temporal lobe epilepsy reproducibility and relation to outcome. Neurology, 74, 1776-1784. 
Bloudek, L. M., Spackman, D. E., Blankenburg, M., \& Sullivan, S. D. (2011). Review and meta-analysis of biomarkers and diagnostic imaging in Alzheimer's disease. Journal of Alzheimer's Disease, 26, 627-645.

Bohanna, I., Georgiou-Karistianis, N., Hannan, A. J., \& Egan, G. F. (2008). Magnetic resonance imaging as an approach towards identifying neuropathological biomarkers for Huntington's disease. Brain Research Reviews, 58, 209-225.

Booij, J., Tissingh, G., Boer, G. J., Speelman, J. D., Stoof, J. C., Janssen, A. G., ... Van Royen, E. A. (1997). (123I) FP-CIT SPECT shows a pronounced decline of striatal dopamine transporter labelling in early and advanced Parkinson's disease. Journal of Neurology, Neurosurgery \& Psychiatry, 62, 133-140.

Drysdale, A. T., Grosenick, L., Downar, J., Dunlop, K., Mansouri, F., Meng, Y., ... Liston, C. (2017). Resting-state connectivity biomarkers define neurophysiological subtypes of depression. Nature Medicine, 23, 28-38.

Dukart, J., Holiga, Š., Chatham, C., Hawkins, P., Forsyth, A., McMillan, R., ... Sambataro, F. (2018). Cerebral blood flow predicts differential neurotransmitter activity. Scientific Reports, 8, 4074.

Feng, J., Cai, X., Zhao, J., \& Yan, Z. (2001). Serotonin receptors modulate GABAA receptor channels through activation of anchored protein kinase $C$ in prefrontal cortical neurons. The Journal of Neuroscience, 21, 6502-6511.

Friston, K. J., Holmes, A. P., Worsley, K. J., Poline, J.-P., Frith, C. D., \& Frackowiak, R. S. (1994). Statistical parametric maps in functional imaging: A general linear approach. Human Brain Mapping, 2, 189-210.

Gómez, F. J. G., Huertas, I., Ramírez, J. A. L., \& Solís, D. G. (2018). Elaboración de una plantilla de SPM para la normalización de imágenes de PET con 18F-DOPA. Imagen Diagnóstica, 9, 23-25.

Good, C. D., Johnsrude, I. S., Ashburner, J., Henson, R. N., Friston, K. J., \& Frackowiak, R. S. (2001). A voxel-based morphometric study of ageing in 465 normal adult human brains. Neurolmage, 14, 21-36.

Hawkins, P., Wood, T. C., Vernon, A. C., Bertolino, A., Sambataro, F., Dukart, J., ... Mehta, M. A. (2018). An investigation of regional cerebral blood flow and tissue structure changes after acute administration of antipsychotics in healthy male volunteers. Human Brain Mapping, 39, 319-331.

Hesse, S., Becker, G.-A., Rullmann, M., Bresch, A., Luthardt, J., Hankir, M. K., ... Sabri, O. (2017). Central noradrenaline transporter availability in highly obese, non-depressed individuals. European Journal of Nuclear Medicine and Molecular Imaging, 44, 1056-1064.

Holiga, S., Hipp, J. F., Chatham, C. H., Garces, P., Spooren, W., Logier D'Ardhuy, X., ... Dukart, J. (2019). Patients with autism spectrum disorders display reproducible functional connectivity alterations. Science Translational Medicine, 11, eaat9223.

Holiga, Š., Sambataro, F., Luzy, C., Greig, G., Sarkar, N., Renken, R. J., ... Dukart, J. (2018). Test-retest reliability of task-based and resting-state blood oxygen level dependence and cerebral blood flow measures. PLoS One, 13, e0206583.

Kaller, S., Rullmann, M., Patt, M., Becker, G.-A., Luthardt, J., Girbardt, J., ... Sabri, O. (2017). Test-retest measurements of dopamine D1-type receptors using simultaneous PET/MRI imaging. European Journal of Nuclear Medicine and Molecular Imaging, 44, 1025-1032.

Lee, M. H., Smyser, C. D., \& Shimony, J. S. (2013). Resting-state fMRI: A review of methods and clinical applications. American Journal of Neuroradiology, 34, 1866-1872.

Lehto, J., Johansson, J., Vuorilehto, L., Luoto, P., Arponen, E., Scheinin, H., ... Scheinin, M. (2015). Sensitivity of (11 C) ORM-13070 to increased extracellular noradrenaline in the CNS-a PET study in human subjects. Psychopharmacology, 232, 4169-4178.

Leuthardt, E. C., Guzman, G., Bandt, S. K., Hacker, C., Vellimana, A. K., Limbrick, D., ... Benzinger, T. L. S. (2018). Integration of resting state functional MRI into clinical practice - a large single institution experience. PLoS One, 13, e0198349. https://www.ncbi.nlm.nih.gov/pmc/ articles/PMC6014724/
Liu, Z., Rolls, E. T., Liu, Z., Zhang, K., Yang, M., Du, J., ... Feng, J. (2019). Brain annotation toolbox: Exploring the functional and genetic associations of neuroimaging results. Bioinformatics, 35, 3771-3778.

Mawlawi, O., Martinez, D., Slifstein, M., Broft, A., Chatterjee, R., Hwang, D.-R., ... Van Heertum, R. (2001). Imaging human mesolimbic dopamine transmission with positron emission tomography: I. accuracy and precision of D2 receptor parameter measurements in ventral striatum. Journal of Cerebral Blood Flow \& Metabolism, 21, 1034-1057.

McCann, U. D., Szabo, Z., Seckin, E., Rosenblatt, P., Mathews, W. B., Ravert, H. T., ... Ricaurte, G. A. (2005). Quantitative PET studies of the serotonin transporter in MDMA users and controls using (11 C) McN5652 and (11 C) DASB. Neuropsychopharmacology, 30, 1741-1750.

Meyer-Lindenberg, A., \& Weinberger, D. R. (2006). Intermediate phenotypes and genetic mechanisms of psychiatric disorders. Nature Reviews Neuroscience, 7, 818-827.

Mikl, M., Mareček, R., Hluštík, P., Pavlicová, M., Drastich, A., Chlebus, P., ... Krupa, P. (2008). Effects of spatial smoothing on fMRI group inferences. Magnetic Resonance Imaging, 26, 490-503.

Pagano, G., Niccolini, F., Fusar-Poli, P., \& Politis, M. (2017). Serotonin transporter in Parkinson's disease: A meta-analysis of positron emission tomography studies. Annals of Neurology, 81, 171-180.

Rizzo, G., Veronese, M., Expert, P., Turkheimer, F. E., \& Bertoldo, A. (2016). MENGA: A new comprehensive tool for the integration of neuroimaging data and the Allen human brain Transcriptome atlas. PLoS One, 11, e0148744. https://www.ncbi.nlm.nih.gov/pmc/articles/PMC4755531/

Savli, M., Bauer, A., Mitterhauser, M., Ding, Y.-S., Hahn, A., Kroll, T., ... Lanzenberger, R. (2012). Normative database of the serotonergic system in healthy subjects using multi-tracer PET. Neurolmage, 63, 447-459.

Schotte, A., Janssen, P. F. M., Gommeren, W., Luyten, W. H. M. L., Van Gompel, P., Lesage, A. S., ... Leysen, J. E. (1996). Risperidone compared with new and reference antipsychotic drugs: in vitro and in vivo receptor binding. Psychopharmacology, 124, 57-73.

Selvaggi, P., Hawkins, P. C. T., Dipasquale, O., Rizzo, G., Bertolino, A., Dukart, J., ... Mehta, M. A. (2018). Increased cerebral blood flow after single dose of antipsychotics in healthy volunteers depends on dopamine D2 receptor density profiles. Neurolmage, 188, 774-784.

Smith, G. S., Price, J. C., Lopresti, B. J., Huang, Y., Simpson, N., Holt, D., ... Nichols, T. (1998). Test-retest variability of serotonin 5-HT2A receptor binding measured with positron emission tomography and (18F) altanserin in the human brain. Synapse, 30, 380-392.

Telesford, Q. K., Morgan, A. R., Hayasaka, S., Simpson, S. L., Barret, W., Kraft, R. A., ... Laurienti, P. J. (2010). Reproducibility of graph metrics in $\mathrm{fMRI}$ networks. Frontiers in Neuroinformatics, 4, 117.

Thompson, P. M., Jahanshad, N., Ching, C. R., Salminen, L. E., Thomopoulos, S. I., Bright, J., ... Bruin, W. B. (2020). ENIGMA and global neuroscience: A decade of large-scale studies of the brain in health and disease across more than 40 countries. Translational Psychiatry, 10, 1-28.

Thompson, P. M., Stein, J. L., Medland, S. E., Hibar, D. P., Vasquez, A. A., Renteria, M. E., ... Franke, B. (2014). The ENIGMA consortium: Largescale collaborative analyses of neuroimaging and genetic data. Brain Imaging and Behavior, 8, 153-182.

Tzourio-Mazoyer, N., Landeau, B., Papathanassiou, D., Crivello, F., Etard, O., Delcroix, N., ... Joliot, M. (2002). Automated anatomical labeling of activations in SPM using a macroscopic anatomical parcellation of the MNI MRI single-subject brain. Neurolmage, 15, 273-289.

Unterholzner, J., Gryglewski, G., Philippe, C., Seiger, R., Pichler, V., Godbersen, G. M., ... Lanzenberger, R. (2020). Topologically guided prioritization of candidate gene transcripts Coexpressed with the 5-HT1A receptor by combining in vivo PET and Allen human brain atlas data. Cerebral Cortex, 30, 3771-3780.

van Erp T. G. M., Hibar D. P., Rasmussen J. M., Glahn D. C., Pearlson G. D., Andreassen O. A., ... Turner J. A. (2016): Subcortical brain volume 
abnormalities in 2028 individuals with schizophrenia and 2540 healthy controls via the ENIGMA consortium. Molecular Psychiatry, 21, 547-553.

\section{SUPPORTING INFORMATION}

Additional supporting information may be found online in the Supporting Information section at the end of this article.
How to cite this article: Dukart J, Holiga S, Rullmann M, et al. JuSpace: A tool for spatial correlation analyses of magnetic resonance imaging data with nuclear imaging derived neurotransmitter maps. Hum Brain Mapp. 2021;42:555-566. https://doi.org/10.1002/hbm.25244 